\title{
QUASI-RETRACTIVE REPRESENTATION OF SOLUTION SETS TO STOCHASTIC INCLUSIONS
}

\author{
MICHAE KISIELEWICZ \\ Technical University, Institute of Mathematics \\ Podgórna 50, 65-246 Zielona Góra, Poland
}

(Received February, 1996; Revised March, 1997)

Continuous dependence and retraction properties of solution sets to
stochastic inclusions $x_{t}-x_{s} \in \int_{s}^{t} F_{\tau}\left(x_{\tau}\right) d \tau+\int_{s}^{t} G_{\tau}\left(x_{\tau}\right) d \omega_{\tau}+$
$\int_{s}^{t} \int_{\mathbb{R}^{n}} H_{\tau, r}\left(x_{\tau}\right) \widetilde{\nu}(d \tau, d r)$ are considered.

Key words: Stochastic Inclusions, Lower Semicontinuous Dependence of Solution Set, Retraction Theorem.

AMS subject classifications: 93E03, 93C30.

\section{Introduction}

Properties of solution sets to stochastic inclusions play a crucial role in stochastic optimal control theory. The first results dealing with this topic are given in the author's paper [4], in which, by rather strong assumptions the weak compactness of the set of all solutions to stochastic inclusions

$$
x_{t}-x_{s} \in \int_{s}^{t} F_{\tau}\left(x_{\tau}\right) d \tau+\int_{s}^{t} G_{\tau}\left(x_{\tau}\right) d w_{\tau}+\int_{s}^{t} \int_{\mathbb{R}^{n}} H_{\tau, r}\left(x_{\tau}\right) \tilde{\nu}(d \tau, d r)
$$

has been obtained. In the present paper, we show that for a given random variable $\lambda$, the solution set $e_{\lambda}$ to an initial value problem

$$
x_{t}-x_{s} \in \int_{s}^{t} F_{\tau}\left(x_{\tau}\right) d \tau+\int_{s}^{t} G_{\tau}\left(x_{\tau}\right) d w_{\tau}+\int_{s}^{t} \int_{\mathbb{R}^{n}} H_{\tau, r}\left(x_{\tau}\right) \tilde{\nu}(d \tau, d r), x_{0}=\lambda,
$$

has quasi-retractive representation. As a result, we obtain lower semicontinuous dependence of solution set $C_{\lambda}$ on an initial date.

We begin with basic notations dealing with set-valued stochastic integrals. Some properties of fixed point sets to subtrajectory integral mappings are investigated. Hence, the main results of this paper readily follow. 


\section{Basic Definitions and Notations}

Let $\left(\Omega, \mathcal{F},\left(\mathcal{F}_{t}\right)_{t}>, P\right)$ be a complete, filtered probability space. Given $T>0$, let $I=$ $[0, T]$ and let $\mathcal{F}_{B}(I)$ denote the Borel $\sigma$-algebra on $I$. We consider set-valued

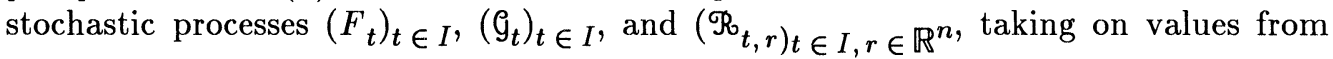
the space $\operatorname{Conv}\left(\mathbb{R}^{n}\right)$ of all nonempty, compact convex subsets of the $n$-dimensional Euclidean space $\mathbb{R}^{n}$. These processes are assumed to be nonanticipative such that $\int_{0}^{T}\left\|F_{t}\right\|^{2} d t<\infty ; \int_{0}^{T}\left\|\mathscr{G}_{t}\right\|^{s} d t<\infty ;$ and $\int_{0}^{T} \int_{\mathbb{R}^{n}}\left\|\mathscr{R}_{t, z}\right\|^{2} d t q(d z)<\infty$, a.s., where $q$ is a measure on a Borel $\sigma$-algebra $\mathfrak{B}^{n}$ of $\mathbb{R}^{n}, A \in \operatorname{Conv}\left(\mathbb{R}^{n}\right)$, and $\|A\|:=$ $\sup \{|a|: a \in A\}$. The space $\operatorname{Conv}\left(\mathbb{R}^{n}\right)$ is endowed with the Hausdorff metric $h$ defined in the usual way (i.e., $h(A, B)=\max \{\bar{h}(A, B), \bar{h}(B, A)\}$, for $A, B \in$ $\operatorname{Conv}\left(\mathbb{R}^{n}\right)$, where $\bar{h}(A, B)=\{\operatorname{dist}(a, B): a \in A\}$ and $\left.\bar{h}(B, A)=\{\operatorname{dist}(b, A): b \in B\}\right)$. $\mathrm{Cl}(X)$ denotes the family of all nonempty closed subsets of a metric space $(X, \rho)$.

Filtered, complete probability spaces $\left(\Omega, \mathscr{F}_{,}\left(\mathscr{F}_{t}\right)_{t>0}, P\right)$ are assumed to satisfy the usual hypotheses: $(i) \mathscr{F}_{0}$ contains all the $P$-null sets of $\mathscr{F}_{;}$and $(i i) \mathscr{F}_{t}=\bigcap_{u}>{ }_{t} \mathcal{F}_{u}$, all $t, 0 \leq t<\infty$. As usual, we shall consider a set $I \times \Omega$ as a measurable space with the product $\sigma$-algebra $\mathscr{B}(I) \otimes \mathscr{F}$.

$\left(X_{t}\right)_{t \in I}$ denotes an $n$-dimensional stochastic process $x$, understood as a function

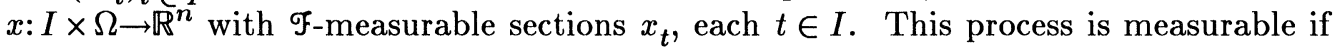

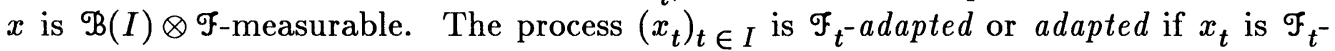
measurable for $t \in I$. Every measurable and adapted process is called nonanticipative.

The Banach spaces $L^{2}\left(\Omega, \mathscr{F}_{t}, P, \mathbb{R}^{n}\right)$ and $L^{2}\left(\Omega, \mathcal{F}, P, \mathbb{R}^{n}\right)$, with the usual norm $\|\cdot\|_{L_{n}^{2}}$, are denoted by $L_{n}^{2}\left(\mathscr{F}_{t}\right)$ and $L_{n}^{2}(F)$, respectively. $\mathcal{M}^{2}\left(\mathcal{F}_{t}\right)$ denotes the family (i.e., equivalence classes) of all $n$-dimensional nonanticipative processes $\left(f_{t}\right)_{t \in I}$ such that $\int_{0}^{T}\left|f_{t}\right|^{2} d t<\infty$, a.s. We shall also consider a subspace $\mathcal{L}_{T}^{2}$ of $\mathcal{M}^{2}\left(\mathcal{F}_{t}\right)$ defined by $\mathcal{L}^{2}=\left\{\left(f_{t}\right)_{t \in 0} \in \mathcal{M}^{2}\left(\mathscr{F}_{t}\right):|f|_{\mathcal{L}^{2}}<\infty\right\}$, with $\quad|f|_{\mathcal{L}^{2}}^{2}=E \int_{0}^{T}\left|f_{t}\right|^{2} d t . \quad$ Finally, $M_{n}\left(\mathscr{F}_{t}\right)$ we denote the space (i.e., equivalence classes) of all $n$-dimensional $\mathscr{F}_{t}$-measurable mappings.

$\left(w_{t}\right)_{t \in I}$ defines a one-dimensional $\mathscr{F}_{t}$-Brownian motion starting at $0 . \quad \nu(t, A)$ denotes a $\mathcal{F}_{t}$-Poisson measure on $I \times \mathscr{B}^{n}$. We define a $\mathcal{F}_{t}$-centered Poisson measure $\widetilde{\nu}(t, A), t \in I, A \in \mathscr{B}^{n}$ by taking $\widetilde{\nu}(t, A)=\nu(t, A)-t q(A), t \in I, A \in \mathfrak{B}^{n}$, where $q$ is a measure on $\mathfrak{B}^{n}$ such that $E \nu(t, B)=t q(B)$ and $q(B)<\infty$ for $B \in \mathfrak{B}_{0}^{n}:=\left\{A \in \mathfrak{B}^{n}\right.$ : $0 \notin \bar{A}\}$.

$\mathcal{M}^{2}\left(\mathscr{F}_{t}, q\right)$ denotes the family (i.e., equivalence classes) of all $\mathscr{B}(I) \otimes \mathscr{F} \otimes \mathscr{B}^{n}$-measurable and $\mathscr{F}_{t^{-}}$-adapted functions $h: I \times \Omega \times \mathbb{R}^{n} \rightarrow \mathbb{R}^{n}$ such that $\int_{0}^{T} \int_{\mathbb{R}^{n}}\left|h_{t, r}\right|^{2} d t q(d r)<$ $\infty$, a.s. Recall, a function $h: I \times \Omega \times \mathbb{R}^{n} \rightarrow \mathbb{R}^{n}$ is said to be $\mathscr{F}_{t}$-adapted or adapted if $h(t, \cdot, r)$ is $\mathscr{F}_{t}$-measurable for every $r \in \mathbb{R}^{n}$ and $t \in I$. Elements of $\mathcal{M}^{2}\left(\mathcal{F}_{t}, q\right)$ will be

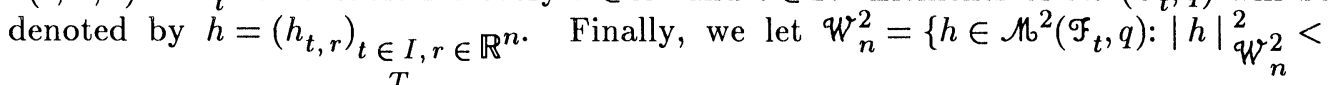
$\infty\}$, where $|h|_{\mathcal{W}_{n}^{2}}^{2}=E \int_{0}^{T} \int_{\mathbb{R}^{n}}\left|h_{t, r}\right|^{2} d t q(d r)$.

Given $f, g \in \mathcal{H}^{2}\left(\mathcal{F}_{t}\right)$ and $h \in \mathcal{M}^{2}\left(\mathcal{F}_{t}, q\right),\left(\int_{0}^{t} f_{\tau} d \tau\right)_{t \in I},\left(\int_{0}^{t} g_{\tau} d w_{\tau}\right) t \in I$, and 
$\left(\int_{0}^{t} \int_{\mathbb{R}^{n}} h_{\tau, r} \tilde{\nu}(d \tau, d r)\right)_{t \in I}$ denote their stochastic integrals with respect to Lebesgue measure on $\mathbb{R}^{+}$, the $\mathscr{F}_{t}$-Brownian motion $\left(w_{t}\right)_{t \in I}$, and the $\mathscr{F}_{t}$-centered Poisson measure $\widetilde{\nu}(t, A), t \in I, A \in \mathfrak{B}^{n}$, respectively. For fixed $t \in I$ and $(f, g, h) \in \mathcal{L}^{2} \times \mathcal{L}^{2} \times$ $W^{2}$,we equate $g_{t}(f)=\int_{0}^{t} f_{\tau} d \tau, g_{t}(g)=\int_{0}^{t} g_{\tau} d w_{\tau}$, and $\mathcal{\sigma}_{t}(h)=\int_{0}^{t} \int_{\mathbb{R}^{n}} h_{\tau, z} \widetilde{\nu}(d \tau, d z)$. $g, g$, and $\mathcal{T}$ denote linear mappings defined by $\mathcal{L}^{2} \ni f \rightarrow\left(g_{t}(f)\right)_{t \in I} \in D, \mathcal{L}^{2} \ni g \rightarrow$ $\left(g_{t}(g)\right)_{t \in I} \in D$, and $W^{2} \ni h \rightarrow\left(\mathcal{\sigma}_{t}(h)\right)_{t \in I} \in D$, respectively. Here, $D$ denotes the family of all $n$-dimensional $\mathcal{F}_{t}$-adapted cádlág (see [7]) processes $\left(x_{t}\right)_{t \in I}$ such that $E \sup _{t \in I}\left|x_{t}\right|^{2}<\infty$. The space $D$ is considered a normed space with norm $\|\xi\|_{\ell}=\left\|\sup _{t \in I}\left|\xi_{t}\right|\right\|_{L_{1}^{2}}$ for $\xi=\left(\xi_{t}\right)_{t \in I} \in D$. It can be verified that $\left(D,\|\cdot\|_{\ell}\right)$
is a Banach space.

Given a measure space $(X, \mathscr{B}, m)$, a set-valued function $\Re: X \rightarrow \mathrm{Cl}\left(\mathbb{R}^{n}\right)$ is said to be $\mathfrak{B}$-measurable if $\{x \in X: \Re(x) \cap C \neq \emptyset\} \in \mathscr{B}$ for every closed set $C \subset \mathbb{R}^{n}$. For such a multifunction, we define subtrajectory integrals as a set $\varphi(\Re)=\{g \in$ $L^{2}\left(X, \mathscr{B}, m, \mathbb{R}^{n}\right): g(x) \in \Re(x)$ a.e. $\}$. We shall assume that the $\mathscr{B}$-measurable, setvalued function $\Re: X \rightarrow \mathrm{Cl}\left(\mathbb{R}^{n}\right)$ is square integrable bounded (i.e., a real-valued mapping $X \ni x \rightarrow\|\Re(x)\| \in \mathbb{R}+$ belongs to $\left.L^{2}(X, \mathfrak{B}, m, \mathbb{R})\right)$.

Let $\mathcal{G}=\left(\mathcal{G}_{t}\right)_{t \in I}$ be a set-valued stochastic process with values in $\left.\mathrm{ClR}^{n}\right)$, (i.e., a

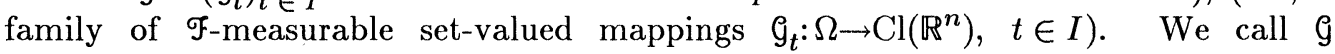

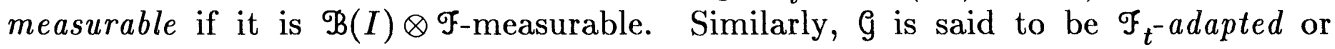
adapted if $g_{t}$ is $\mathscr{F}_{t}$-measurable for each $t \in I$. A measurable and adapted set-valued stochastic process is called nonanticipative.

We shall also consider $\mathfrak{B}(I) \otimes \mathscr{F} \otimes \mathfrak{B}^{n}$-measurable set-valued mappings $\mathscr{B}: I \times \Omega \times$ $\mathbb{R}^{n} \rightarrow \mathrm{Cl}\left(\mathbb{R}^{n}\right)$. These mappings will be denoted by $\left(\mathscr{R}_{t, r}\right)_{t \in I, r \in \mathbb{R}^{n} \text {, and called }}$ measurable set-valued stochastic processes depending on a parameter $r \in \mathbb{R}^{n}$. The process $\mathscr{R}_{0}=\left(\mathscr{R}_{t, z r}\right)_{t \in I, r \in \mathbb{R}^{n}}$ is said to be $\mathscr{F}_{t^{-}}$adapted or adapted if $\mathscr{R}_{t, r}$ is $\mathscr{F}_{t^{-}}$ measurable for each $t \in I$ and $z \in \mathbb{R}^{n}$. We call this process nonanticipative if it is measurable and adapted.

$\mathcal{M}_{s-v}^{2}\left(\mathcal{F}_{t}\right)$ and $\mathcal{M}_{s-v}^{2}\left(\mathcal{F}_{t}, q\right)$ denote families of all nonanticipative set-valued processes $\mathfrak{g}=\left(\mathfrak{G}_{t}\right)_{t \in I}$ and $\mathscr{R}=\left(\mathscr{B}_{t, r}\right)_{t \in I, r \in \mathbb{R}^{n}}$ respectively, such that $\int_{0}^{T}\left\|G_{t}\right\|^{2} d t<\infty$ and $\int_{0}^{T} \int_{\mathbb{R}^{n}}\left\|\mathfrak{B}_{t, r}\right\|^{2} d t q(d r)<\infty$, a.s. From Kuratowski and Ryll-

Nardzewski measurable selection theorem (see [3]) it immediately follows that for every $F, \mathcal{g} \in \mathcal{M}_{s-v}^{2}\left(\mathscr{F}_{t}\right)$ and $\mathscr{R}_{\mathcal{B}} \in \mathcal{M}_{s-v}^{2}\left(\mathscr{F}_{t, q}\right)$, their subtrajectory integrals $\varphi(F):=$ $\left\{f \in \mathcal{M}^{2}\left(\mathcal{F}_{t}\right): f_{t}(\omega) \in F_{t}(\omega), d t \times P\right.$-a.e. $\}, \varphi(\mathcal{G}):=\left\{g \in \mathcal{M}^{2}\left(\mathscr{F}_{t}\right): g_{t}(\omega) \in G_{t}(\omega), d t \times P-\right.$ a.e. $\}$, and $\mathscr{\varphi}_{q}\left(\mathscr{R}_{0}\right):=\left\{h \in \mathcal{H}^{2}\left(\mathscr{F}_{t}, q\right): h_{t, r}(\omega) \in \mathscr{R}_{t, r}(\omega), d t \times P \times q\right.$-a.e. $\}$ are nonempty. Indeed, we let $\Sigma=\left\{Z \in \mathscr{B}(I) \otimes \mathscr{F}_{:} Z_{t} \in \mathcal{F}_{t}\right.$, each $\left.t \in I\right\}$, where $Z_{t}$ denotes a section of $Z$ determined by $t \in I . \quad \Sigma$ is a $\sigma$-algebra on $I \times \Omega$, and a function $f: I \times \Omega \rightarrow \mathbb{R}^{n}$ (a multifunction $\left.F: I \times \Omega \rightarrow \mathrm{Cl}\left(\mathbb{R}^{n}\right)\right)$ is nonanticipative if and only if it is $\Sigma$-measurable. Therefore, by Kuratowski and Ryll-Nardzewski measurable selection theorem, every nonanticipative set-valued function admits a nonanticipative selector. It is clear that for $F \in \mathcal{M}_{s-v}^{2}\left(\mathcal{F}_{t}\right)$, such selectors belong to $\mathcal{M}^{2}\left(\mathscr{F}_{t}\right)$. Similarly, we define on $I \times \Omega \times$ $\mathbb{R}^{n}$ a $\sigma$-algebra $\Sigma=\left\{Z \in \mathfrak{B}(I) \otimes \mathscr{F} \otimes \mathscr{B}^{n}: Z_{t}^{u} \in \mathcal{F}_{t}\right.$, each $t \in I$ and $\left.u \in \mathbb{R}^{n}\right\}$, where $Z_{t}^{u}=\left(Z^{u}\right)_{t}$, and $Z^{u}$ denotes a section of $Z$ determined by $u \in \mathbb{R}^{n}$. The foregoing arguments can be repeated to obtain the above result for nonanticipative, set-valued 
processes depending on a parameter $r \in \mathbb{R}^{n}$.

It can be verified (see $[2,3]$ ) that for given $F=\left(\mathscr{F}_{t}\right)_{t \in I} \in \mathcal{M}_{s-v}^{2}\left(\mathcal{F}_{t}\right)$,

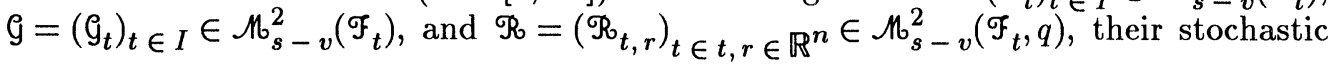
integrals are defined as families $\left(\int_{0}^{t} F_{\tau} d \tau\right)_{t \in I},\left(\int_{0}^{t} g_{\tau} d w_{\tau}\right)_{t \in I}, \quad$ and $\left(\int_{0}^{t} \int_{\mathbb{R}^{n}} \mathscr{R}_{\tau, z} \widetilde{\nu}(d \tau, d z)\right)_{t \in I}$ of the subsets of $M\left(\mathscr{F}_{t}\right)$, of the form $\int_{0}^{t} \mathscr{F}_{\tau} d \tau=\left\{\int_{0}^{t} f_{\tau} d \tau\right.$ : $f \in \mathcal{Y}(F)\}, \quad \int_{0}^{t} \mathfrak{g}_{\tau} d w_{\tau}=\left\{\int_{0}^{t} g_{\tau} d w_{\tau}: g \in \mathcal{Y}^{2}(\mathfrak{G})\right\} \quad$ and $\left.\quad \int_{0}^{t} \int_{\mathbb{R}^{n}} h_{\tau, z} \widetilde{\nu}(d \tau, d z): h \in \mathcal{Y}_{q}\left(\mathscr{B}_{0}\right)\right\}$. Given $0 \leq \alpha<\beta<\infty$, we also define $\int_{\alpha}^{\beta} F_{s} d s:=\left\{\int_{\alpha}^{\beta} f_{s} d s: f \in \varphi^{p}(F)\right\}, \int_{\alpha}^{\beta} \varrho_{s} d w_{s}:=$ $\left\{\int_{\alpha}^{\beta} g_{s} d w_{s}: g \in \varphi^{2}(\mathcal{g})\right\}$, and $\int_{\alpha}^{\beta} \int_{\mathbb{R}^{n}} \mathscr{R}_{s, r} \widetilde{\nu}(d s, d z):=\left\{\int_{\alpha}^{\beta} \int_{\mathbb{R}^{n}} h_{s, r} \widetilde{\nu}(d s, d z): h \in \varphi_{q}\left(\mathscr{R}_{0}\right)\right\}$.

The following selection property of set-valued stochastic integrals has been obtained in [5]:

Proposition 1. Let $F, \mathcal{G} \in \mathcal{M}_{s-v}^{2}\left(\mathscr{F}_{t}\right), \Re_{\mathcal{B}} \in \mathcal{M}_{s-v}^{2}\left(\mathscr{F}_{t}, q\right)$, and $\left(x_{t}\right)_{t \in I} \in D$. Then:

$$
x_{t}-x_{s} \in \int_{s}^{t} F_{\tau} d \tau+\int_{s}^{t} G_{\tau} d w_{\tau}+\int_{s}^{t} \int_{\mathbb{R}^{n}} \mathscr{R}_{\tau, r} \tilde{\nu}(d \tau, d r)
$$

for $0 \leq s \leq t \leq T$ if and only if there exists $(f, g, h) \in \mathcal{Y}(\mathscr{F}) \times \mathscr{Y}(\mathcal{G}) \times \mathcal{Y}_{q}\left(\mathscr{P}_{\mathcal{B}}\right)$ such that

for $t \in I$.

$$
x_{t}=\int_{0}^{t} f_{\tau} d \tau+\int_{0}^{t} g_{\tau} d w_{\tau}+\int_{0}^{t} \int_{\mathbb{R}^{n}} h_{\tau, r} \widetilde{\nu}(d \tau, d r)
$$

\section{Stochastic Inclusions and Subtrajectory Integrals Depending on Parameters}

Let

$$
\begin{gathered}
F=\left\{\left(F_{t}(x)\right)_{t \in I}: x \in \mathbb{R}^{n}\right\}, G=\left\{\left(G_{t}(x)\right)_{t \in I}: x \in \mathbb{R}^{n}\right\}, \\
\text { and } H=\left\{\left(H_{t, r}(x)\right)_{\left.t \in I, r \in \mathbb{R}^{n}: x \in \mathbb{R}^{n}\right\} .}\right.
\end{gathered}
$$

Assume $F, G$, and $H$ are such that $\left(F_{t}(x)\right)_{t \in I} \in \mathcal{M}_{s-v}^{p}\left(\mathscr{F}_{t}\right), \quad\left(G_{t}(x)\right)_{t \in I} \in$

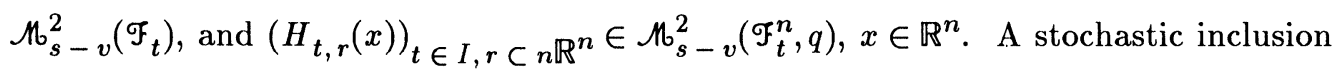
denoted by $S I(F, G, H)$, corresponding to the aforementioned $F, G$, and $H$ is the relation:

$$
x_{t}-x_{s} \in \int_{s}^{t} F_{\tau}\left(x_{\tau}\right) d \tau+\int_{s}^{t} G_{\tau}\left(x_{\tau}\right) d w_{\tau}+\int_{s}^{t} \int_{\mathbb{R}^{n}} H_{\tau, r}\left(x_{\tau}\right) \tilde{\nu}(d \tau, d z),
$$


which is satisfied for every $0 \leq s<t<T$ by a stochastic process $x=\left(x_{t}\right)_{t \in I} \in D$ such that $F \circ x \in \mathcal{M}_{s-v}^{2}\left(\mathscr{F}_{t}\right), \bar{G} \circ x \in \mathcal{M}_{s-v}^{2}\left(\mathcal{F}_{t}\right)$, and $H \circ x \in \mathcal{M}_{s-v}^{2}\left(\mathscr{F}_{t}, q\right)$, where

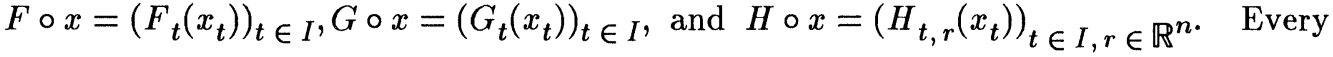
stochastic process $\left(x_{t}\right)_{t \in I} \in D$, satisfying conditions mentioned above, is said to be a global solution to $S I(F, G, H)$. Given $\lambda \in L_{n}^{2}\left(\mathcal{F}_{0}\right)$ we shall consider $S I(F, G, H)$ together with an initial value condition $x_{0}=\lambda$. This type of initial value problem will be denoted by $S I_{\lambda}(F, G, H)$.

We shall assume that $F, G$, and $H$ satisfy the following condition:

$\left(\mathcal{A}_{1}\right): \quad$ (i) $\quad F=\left\{\left(F_{t}(x)\right)_{t \in I}: x \in \mathbb{R}^{n}\right\}, G=\left\{\left(G_{t}(x)\right)_{t \in I}: x \in \mathbb{R}^{n}\right.$, and $H=$

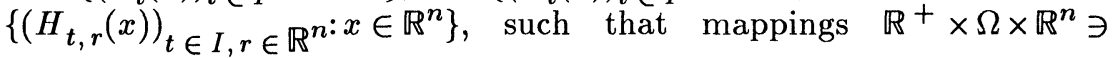
$(t, \omega, x) \rightarrow F_{t}(x)(\omega) \in \operatorname{Conv}\left(\mathbb{R}^{n}\right), I \times \Omega \times \mathbb{R}^{n} \ni(t, \omega, x) \rightarrow G_{t}(x)(\omega) \in$ $\operatorname{conv}\left(\mathbb{R}^{n}\right)$, and $I \times \Omega \times \mathbb{R}^{n} \times \mathbb{R}^{n} \ni(t, \omega, r, x) \rightarrow H_{t, r}(x)(\omega) \in \operatorname{Conv}\left(\mathbb{R}^{n}\right)$ are $\Sigma \otimes \mathscr{B}^{n}$ and $\widetilde{\Sigma} \otimes \mathscr{B}^{n}$-measurable, respectively;

(ii) $\left(F_{t}(x)\right)_{t \in I},\left(G_{t}(x)\right)_{t \in I}$, and $\left(H_{x, r}(x)\right)_{t \in I, r \in \mathbb{R}^{n}}$ are uniformly square

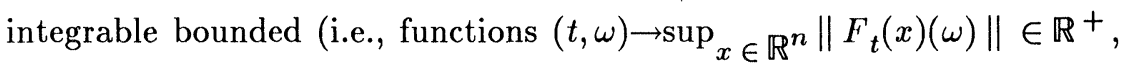

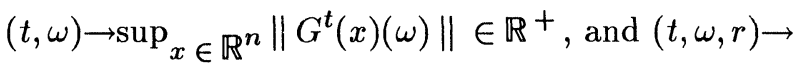
$\left.\sup _{x \in \mathbb{R}^{n}}\left\|H_{t, r}(x)(\omega)\right\| \in \mathbb{R}^{+}\right)$are square integrable on $\mathbb{R}^{+} \times \Omega$ and $\mathbb{R}^{+} \times \Omega \times \mathbb{R}^{n}$, respectively.

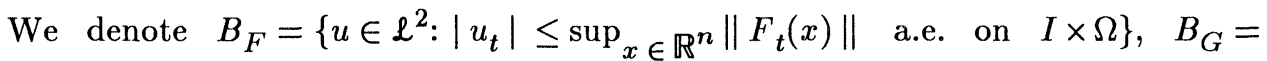

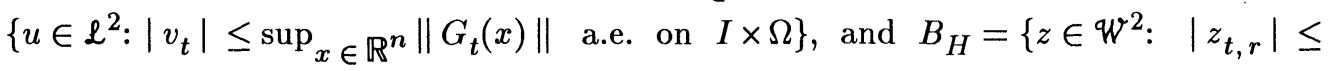

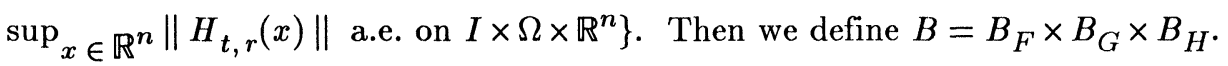

Corollary 1. If $F, G, H$ satisfy $\left(\mathcal{A}_{1}\right)$, then $B$ is a nonempty convex and weakly compact subset of $\mathcal{L}^{2} \times \mathcal{L}^{2} \times W^{2}$. Moreover, for every $\left(x_{t}\right)_{t \in I} \in D$, one has $F \circ x$, $G \circ x \in \mathcal{M}_{s-v}^{2}\left(\mathcal{F}_{t}\right)$ and $H \circ x \in \mathcal{M}_{s-v}^{2}\left(\mathcal{F}_{t}^{n}, q\right)$

Let $\Phi$ be a linear mapping on $\ell^{2} \times \ell^{2} \times W^{2}$ defined by $\Phi=g+g+\sigma$, (i.e., $\Phi(f, g, h)=\left(g_{t} f+g_{t} g+\mathcal{T}_{g} h\right)_{t \in I}$ for $\left.(f, g, h) \in \mathcal{L}^{2} \times \mathcal{L}^{2} \times \mathcal{W}^{2}\right)$. For fixed $\lambda \in L_{n}^{2}\left(\mathcal{F}_{0}\right)$, $\Phi^{\lambda}$ denotes an affine mapping on $\mathcal{L}^{2} \times \mathcal{L}^{2} \times \mathcal{W}^{2}$ defined by $\Phi^{\lambda}(u, v, r)=\lambda+\Phi(f, g, h)$ for $(f, g, h) \in \mathcal{L}^{2} \times \mathcal{L}^{2} \times \mathcal{W}^{2}$. Given $F, G$, and $H$ and $\lambda \in L_{n}^{2}\left(\mathcal{F}_{0}\right)$, we set

$$
\mathfrak{H}_{\lambda}(x)=\Phi^{\lambda}\left(\varphi(F \circ x) \times \varphi(G \circ x) \times \mathcal{Y}_{q}(H \circ x)\right)
$$

for $x=\left(x_{t}\right)_{t \in I} \in D$. It can be verified (see $[4,5]$ ) that for every $x \in D, \mathfrak{H}_{\lambda}(x)$ is a convex, weakly compact subset of $D$. $\mathcal{J}_{\lambda}$ denotes a set-valued mapping $D \ni x \rightarrow$ $\mathcal{H}_{\lambda}(x) \subset D$. From Proposition 1, it immediately follows that for every $\lambda \in L_{n}^{2}\left(\mathscr{F}_{0}\right)$, and $F, G$, and $H$ satisfying condition $\left(\mathcal{A}_{1}\right), x \in D$ is a solution to $S I_{\lambda}(F, G, h)$ if and only if $x$ is a fixed point to $\mathfrak{J}_{\boldsymbol{H}}$.

Suppose $F=\left\{\left(F_{t}(x)\right)_{t \in I}: x \in \mathbb{R}^{n}\right\}, G=\left\{\left(G_{t}(x)\right)_{t \in I}: x \in \mathbb{R}^{n}\right\}$, and

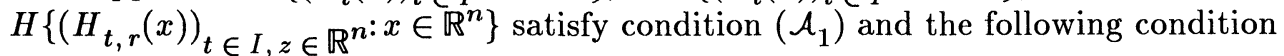

$\left(\mathcal{A}_{2}\right)$ There are $k_{F}, k_{G} \in L_{1}^{2}(\mathscr{B}(I))$ and $m \in L_{1}^{2}\left(\mathscr{B}(I) \times \mathscr{B}^{n}\right)$ such that $h\left(F_{t}\left(x_{2}\right), F_{t}\left(x_{1}\right)\right) \leq k_{F}(t)\left|x_{2}-x_{1}\right|, h\left(G_{t}\left(x_{2}\right), G_{t}\left(x_{1}\right)\right) \leq k_{G}(t)\left|x_{2}-x_{1}\right|$, and $h\left(H_{t, r}\left(x_{2}\right), H_{t, r}\left(x_{1}\right)\right) \leq m(t, r)\left|x_{2}-x_{1}\right|$ a.s., each $t \in I$ and $x_{1}, x_{2} \in \mathbb{R}^{n}$.

Consider for fixed $\lambda \in L_{n}^{2}\left(\mathscr{F}_{0}\right)$ a subtrajectory integrals mapping $S_{\lambda}$ defined by: 


$$
\left.S_{\lambda}(u, v, r)=\varphi\left(F \circ \Phi^{\lambda}(u, v, r)\right) \times \varphi\left(G \circ \Phi^{\lambda}(u, v, r)\right) \times \varphi_{q}\left(H \circ \Phi^{\lambda} u, v, r\right)\right)
$$

for $(u, v, r) \in \mathcal{L}^{2} \times \mathcal{L}^{2} \times \mathcal{W}^{2}$. It is clear that for $\lambda \in L_{n}^{2}\left(\mathscr{F}_{0}\right),(u, v, r) \in \mathcal{L}^{2} \times \mathcal{L}^{2} \times \mathcal{W}^{2}$; and $F, G$, and $H$ satisfying condition $\left(\mathcal{A}_{1}\right)$, one has $S_{\lambda}(u, v, r) \in \mathrm{Cl}\left(\mathcal{L}^{2} \times \mathcal{L}^{2} \mathcal{W}^{2}\right)$. We shall show that if condition $\left(\mathcal{A}_{2}\right)$ is satisfied, then it is possible to renorm a space $\mathcal{L}^{2} \times \mathcal{L}^{2} \times \mathcal{W}^{2}$ by an equivalent norm $\|\cdot\|$ such that $S_{\lambda}(\cdot)$ is a contraction from $\left(\mathcal{L}^{2} \times \mathcal{L}^{2} \times W^{2},\|\cdot\|\right)$ into $\left(\mathrm{Cl}\left(\mathcal{L}^{2} \times \mathcal{L}^{2} \times \mathcal{W}^{2}\right), \ell\right)$, where $\ell$ is the Hausdorff metric induced by $\|\cdot\| \cdot \mathrm{A}$ similar result is also true for $S .(u, v, r): L_{n}^{2}\left(\mathscr{F}_{0}\right) \ni \lambda \rightarrow$ $S_{\lambda}(u, v, z) \in \mathrm{Cl}\left(\mathcal{L}^{2} \times \mathcal{L}^{2} \times \mathscr{W}^{2}\right)$. Observe that a norm $\|\cdot\|$ is defined by $\|(u, v, r)\|=\max \left(\|u\|_{\mathcal{L}^{2}},\|v\|_{\mathcal{L}^{2}},\|z\|_{W^{2}}\right)$, where $\|\cdot\|_{\mathcal{L}^{2}}$ and $\|\cdot\|_{\mathcal{W}^{2}}$ are appropriate norms on $\mathcal{L}^{2}$ and $\mathscr{W}^{2}$ equivalent to $|\cdot|_{\ell^{2}}$ and $|\cdot|_{\mathscr{W}^{2}}$ defined above.

Finally, observe that for every $A, \widetilde{A}, B, \widetilde{B} \in \mathrm{Cl}\left(\mathcal{L}^{2}\right)$ and $C, \widetilde{C} \in \mathrm{Cl}\left(\mathcal{W}^{2}\right)$ one has:

$$
\ell(A \times B \times C, \tilde{A} \times \widetilde{B} \times \widetilde{C}) \leq \max \left\{\ell_{\mathcal{L}^{2}}(A, \tilde{A}), \ell_{\mathcal{L}^{2}}(B, \widetilde{B}), \ell_{\mathcal{W}^{2}}(C, \widetilde{C})\right\}
$$

where $\ell_{\ell^{2}}$ and $\ell_{W^{2}}$ are Hausdorff metrics on $\mathrm{Cl}\left(\mathcal{L}^{2}\right)$ and $\mathrm{Cl}\left(\mathcal{W}^{2}\right)$ induced by the norms $\|\cdot\|_{L^{2}}$ and $\|\cdot\|_{W^{2}}$, respectively.

Proposition 2. Suppose $F, G$, and $H$ satisfy $\left(\mathcal{A}_{1}\right)$ and $\left(\mathcal{A}_{2}\right)$. For every $L>0$, there are norms $\|\cdot\|_{\mathcal{L}^{2}}$ and $\|\cdot\|_{W^{2}}$ on $\mathcal{L}^{2}$ and $W^{2}$ equivalent to $|\cdot|_{\mathcal{L}^{2}}$ and $\left.1 \cdot\right|_{w^{2}}$, respectively, such that:

$$
\begin{gathered}
\ell_{\mathcal{L}_{2}}\left(S\left(F \circ \Phi^{\lambda}(u, v, r)\right), S\left(F \circ \Phi^{\lambda}(\tilde{u}, \tilde{v}, \tilde{z})\right)\right. \\
\leq L \max \left(\|u-\tilde{u}\|_{\mathcal{L}^{2}},\|v-\tilde{v}\|_{\mathcal{L}^{2}},\|z=\tilde{z}\|_{W^{2}}\right), \\
\ell_{\mathcal{L}^{2}}\left(S\left(G \circ \Phi^{\lambda}(u, v, r)\right), S\left(G \circ \Phi^{\lambda}(\tilde{u}, \tilde{v}, \tilde{z})\right)\right. \\
\leq L \max \left(\|u-\tilde{u}\|_{\mathcal{L}^{2}},\|v-\tilde{v}\|_{\mathcal{L}^{2}},\|z-\tilde{z}\|_{W^{2}}\right), \\
\quad \ell_{\mathcal{L}^{2}}\left(S_{q}\left(H \circ \Phi^{\lambda}(u, v, r)\right), S_{q}\left(H \circ \Phi^{\lambda}(\tilde{u}, \tilde{v}, \tilde{z})\right)\right. \\
\leq L \max \left(\|u-\tilde{u}\|_{\mathcal{L}^{2}},\|v-\tilde{v}\|_{\mathcal{L}^{2}},\|z-\tilde{z}\|_{W^{2}}\right),
\end{gathered}
$$

and

for $(u, v, r),(\tilde{u}, \widetilde{v}, \widetilde{z}) \in \mathcal{L}^{2} \times \mathcal{L}^{2} \times W^{2}$.

Proof. Let $L>0$ be given and fix $(u, v, z),(\tilde{u}, \tilde{v}, \tilde{z}) \in \mathcal{L}^{2} \times \mathcal{L}^{2} \times \mathcal{W}^{2}$. For every $f \in S\left(F \circ \Phi_{t}^{\lambda}(u, v, z)\right)$, there is $\tilde{f} \in S\left(F \circ \Phi^{\lambda}(\tilde{u}, \tilde{v}, \tilde{z})\right)$ such that:

$$
\begin{aligned}
& \left.\mid f_{t}-\tilde{f}_{t}\right) \leq h\left(F_{t}\left(\Phi_{t}^{\lambda}(u, v, z)\right), F_{t}\left(\Phi_{t}^{\lambda}(\tilde{u}, \tilde{v}, \tilde{z})\right) \leq k_{F}(t)\left|\Phi_{t}^{\lambda}(u, v, z)-\Phi_{t}^{\lambda}(\tilde{u}, \tilde{v}, \tilde{z})\right|\right. \\
& \leq k_{F}(t)\left\{\int_{0}^{t}\left|u_{r}-\tilde{u}_{r}\right| d \tau+\left|\int_{0}^{t}\left[v_{r}-\tilde{v}_{r}\right] d \omega_{\tau}\right|+\left|\int_{0}^{t} \int_{\mathbb{R}^{m}}\left[z_{\tau, r}-\tilde{z}_{\tau, r}\right] \tilde{v}(d \tau, d r)\right|\right\}
\end{aligned}
$$

a.s., each $t \in I$. Similarly, for every $g_{\sim} \in S\left(G \circ \Phi^{\lambda}(u, v, z)\right)$ and $h \in S_{q}\left(H \circ \Phi^{\lambda}(u, v, z)\right)$, there are $\tilde{g} \in S\left(G \circ \Phi^{\lambda}(\widetilde{u}, \widetilde{v}, \tilde{z})\right)$ and $\tilde{h} \in S_{q}\left(H \circ \Phi^{\lambda}(\tilde{u}, \widetilde{v}, \widetilde{z})\right)$ such that: 
and

$$
\begin{gathered}
\left|g_{t}-\tilde{g}_{t}\right| \\
\leq k_{G}(t)\left\{\int_{0}^{t}\left|u_{\tau}-\tilde{u}_{\tau}\right| d \tau+\left|\int_{0}^{t}\left[v_{\tau}-\tilde{v}_{\tau}\right] d \omega_{\tau}\right|+\left|\int_{0}^{t} \int_{\mathbb{R}^{m}}\left[z_{\tau, r}-\tilde{z}_{\tau, r}\right] \tilde{v}(d \tau, d r)\right|\right\} \\
\leq m(t, r)\left\{\int_{0}^{t}\left|u_{r}-\tilde{u}_{r}\right| d \tau+\left|\int_{0}^{t}\left[v_{r}-\tilde{v}_{r}\right] d \omega_{\tau}\right|+\left|\int_{0}^{t} \int_{\mathbb{R}^{m}}\left[z_{\tau, r}-\tilde{z}_{\tau, r}\right] \tilde{v}(d \tau, d r)\right|\right\}
\end{gathered}
$$

a.s., each $t \in I$. Let:

$$
\gamma=\max \left\{(3 / L)^{2} T,(3 / L)^{2}\right\}, k^{2}(t)=\max \left\{k_{F}^{2}(t), k_{G}^{2}(t), \int_{\mathbb{R}^{m}} m^{2}(t, r) q(d r)\right\},
$$

and $\mathscr{K}(t)=\int_{0}^{t} k^{2}(\tau) d \tau$ for $t \in I$. Let us renorm $\mathcal{L}^{2}$ and $\mathscr{W}^{2}$ with equivalent norms $\|\cdot\|_{L^{2}}$ and $\|\cdot\|_{W^{2}}$ defined by:

and

$$
\|u\|_{\mathcal{L}^{2}}=\left(E \int_{0}^{T} e^{-\gamma \mathscr{W}(t)}\left|u_{t}\right|^{2} d t\right)^{1 / 2}
$$

$$
\|z\|_{\mathcal{W}^{2}}=\left(E \int_{0}^{T} \int_{\mathbb{R}^{m}} e^{-\gamma \mathscr{K}(t)}\left|z_{t, r}\right|^{2} q(d r) d t\right)^{1 / 2}
$$

for $u \in \mathcal{L}^{2}$ and $z \in W^{2}$. We obtain:

$$
\begin{aligned}
\|f-\tilde{f}\|_{\mathcal{L}^{2}} \leq\left(E \int_{0}^{T} k^{2}(t) e^{-\gamma \mathscr{K}(t)}\left[\int_{0}^{t}\left|u_{r}-\tilde{u}_{r}\right| d \tau\right]^{2} d t\right)^{1 / 2} \\
+\left(E \int_{0}^{T} k^{2}(t) e^{-\gamma \mathscr{G}(t)}\left|\int_{0}^{t}\left[v_{\tau}-\tilde{v}_{\tau}\right] d \omega_{\tau}\right|^{2} d t\right)^{1 / 2} \\
+\left(E \int_{0}^{T} k^{2}(t) e^{-\gamma \mathscr{G}(t)} \mid \int_{0}^{t} \int_{\mathbb{R}^{m}}^{t}\left[z_{\tau, r}-\left.\tilde{z}_{\tau, r} \tilde{v}(d \tau, d r)\right|^{2} d t\right)^{1 / 2} .\right.
\end{aligned}
$$

We have:

$$
E \int_{0}^{T} k^{2}(t) e^{-\gamma \mathscr{K}(t)}\left[\int_{0}^{t}\left|u_{\tau}-\widetilde{u}_{\tau}\right| d \tau\right]^{2} d t
$$


and

$$
\begin{gathered}
\left|g_{t}-\tilde{g}_{t}\right| \\
\leq k_{G}(t)\left\{\int_{0}^{t}\left|u_{\tau}-\tilde{u}_{\tau}\right| d \tau+\left|\int_{0}^{t}\left[v_{\tau}-\tilde{v}_{\tau}\right] d \omega_{\tau}\right|+\left|\int_{0}^{t} \int_{\mathbb{R}^{m}}\left[z_{\tau, r}-\tilde{z}_{\tau, r}\right] \tilde{v}(d \tau, d r)\right|\right\}
\end{gathered}
$$

$$
\left|h_{t, r}-\tilde{h}_{t, r}\right|
$$

$$
\leq m(t, r)\left\{\int_{0}^{t}\left|u_{r}-\tilde{u}_{r}\right| d \tau+\left|\int_{0}^{t}\left[v_{r}-\tilde{v}_{r}\right] d \omega_{\tau}\right|+\left|\int_{0}^{t} \int_{\mathbb{R}^{m}}\left[z_{\tau, r}-\widetilde{z}_{\tau, r}\right] \tilde{v}(d \tau, d r)\right|\right\}
$$

a.s., each $t \in I$. Let:

$$
\gamma=\max \left\{(3 / L)^{2} T,(3 / L)^{2}\right\}, k^{2}(t)=\max \left\{k_{F}^{2}(t), k_{G}^{2}(t), \int_{\mathbb{R}^{m}} m^{2}(t, r) q(d r)\right\},
$$

and $\mathscr{K}(t)=\int_{0}^{t} k^{2}(\tau) d \tau$ for $t \in I$. Let us renorm $\mathcal{L}^{2}$ and $\mathscr{W}^{2}$ with equivalent norms $\|\cdot\|_{\mathcal{L}^{2}}$ and $\|\cdot\|_{W^{2}}$ defined by:

and

$$
\|u\|_{\mathcal{L}^{2}}=\left(E \int_{0}^{T} e^{-\gamma \mathscr{G}(t)}\left|u_{t}\right|^{2} d t\right)^{1 / 2}
$$

$$
\|z\|_{\mathcal{W}^{2}}=\left(E \int_{0}^{T} \int_{\mathbb{R}^{m}} e^{-\gamma \mathscr{W}(t)}\left|z_{t, r}\right|^{2} q(d r) d t\right)^{1 / 2}
$$

for $u \in \mathcal{L}^{2}$ and $z \in W^{2}$. We obtain:

$$
\begin{aligned}
& \|f-\tilde{f}\|_{\mathcal{L}^{2}} \leq\left(E \int_{0}^{T} k^{2}(t) e^{-\gamma \mathscr{G}(t)}\left[\int_{0}^{t}\left|u_{r}-\tilde{u}_{r}\right| d \tau\right]^{2} d t\right)^{1 / 2} \\
& +\left(E \int_{0}^{T} k^{2}(t) e^{-\gamma \mathscr{G}(t)}\left|\int_{0}^{t}\left[v_{\tau}-\widetilde{v}_{\tau}\right] d \omega_{\tau}\right|^{2} d t\right)^{1 / 2} \\
& +\left(E \int_{0}^{T} k^{2}(t) e^{-\gamma \mathscr{G}(t)}\left|\int_{0}^{t} \int_{\mathbb{R}^{m}}\left[z_{\tau, r}-\tilde{z}_{\tau, r}\right] \widetilde{v}(d \tau, d r)\right|^{2} d t\right)^{1 / 2} .
\end{aligned}
$$

We have:

$$
E \int_{0}^{T} k^{2}(t) e^{-\gamma \mathscr{K}(t)}\left[\int_{0}^{t}\left|u_{\tau}-\tilde{u}_{\tau}\right| d \tau\right]^{2} d t
$$


Therefore,

$$
\begin{gathered}
\ell_{\mathcal{L}^{2}}\left(S\left(F \circ \Phi^{\lambda}(u, v, z)\right), S\left(F \circ \Phi^{\lambda}(\tilde{u}, \tilde{v}, \tilde{z})\right),\right. \\
\leq L \max \left(\|u-\tilde{u}\|_{\mathcal{L}^{2}},\|v-\tilde{v}\|_{\mathcal{L}^{2}},\|z-\tilde{z}\|_{W^{2}} .\right.
\end{gathered}
$$

Similarly,

$$
\begin{gathered}
\quad \ell_{\mathcal{L}^{2}}\left(S\left(G \circ \Phi^{\lambda}(u, v, z)\right), S\left(G \circ \Phi^{\lambda}(\tilde{u}, \widetilde{v}, \tilde{z})\right),\right. \\
\leq L \max \left(\|u-\tilde{u}\|_{\mathcal{L}^{2}},\|v-\tilde{v}\|_{\mathcal{L}^{2}},\|z-\tilde{z}\|_{W^{2}} \cdot\right.
\end{gathered}
$$

Finally,

$$
\begin{gathered}
\leq \mid\|h-\tilde{h}\| W^{2} \\
\left.\leq\left.\left|E \int_{0}^{T} e^{-\gamma \mathscr{K}(t)} \int_{\mathbb{R}^{m}} m^{2}(t, r)\right| \int_{0}^{t}\left[u_{\tau}-\tilde{u}_{\tau}\right] d \tau\right|^{2} q(d \tau) d t\right)^{1 / 2} \\
+\left(E \int_{0}^{T} e^{-\gamma \mathscr{K}(t)} \int_{\mathbb{R}^{m}} m^{2}(t, r)\left|\int_{0}^{t}\left[v_{\tau}-\tilde{v}_{\tau}\right] d \omega_{\tau}\right|^{2} q(d \tau) d t\right)^{1 / 2} \\
+\left(E \int_{0}^{T} e^{-\gamma \mathscr{K}(t) \int_{\mathbb{R}^{m}} m^{2}(t, r)}\left|\int_{0}^{t} \int_{\mathbb{R}^{m}}\left[z_{\tau, r}-\tilde{z}_{\tau, r}\right] \nu(d \tau, d r)\right|^{2} q(d \tau) d t\right)^{1 / 2} .
\end{gathered}
$$

Similarly, as above,

$$
\begin{aligned}
& E \int_{0}^{T} e^{-\gamma \mathscr{K}(t)} \int_{\mathbb{R}^{m}} m^{2}(t, r)\left|\int_{0}^{t}\left[u_{\tau}-\tilde{u}_{\tau}\right] d \tau\right|^{2} q(d \tau) d t \\
& \leq T E \int_{0}^{T} e^{-\gamma \mathscr{K}(t)} \int_{0}^{t}\left|u_{\tau}-\tilde{u}_{\tau}\right|^{2} d \tau d t \leq(L / 3)^{2}\|u-\tilde{u}\|_{\mathcal{L}^{2}}^{2}, \\
& E \int_{0}^{T} e^{-\gamma \mathscr{G}(t)} \int_{\mathbb{R}^{m}} m^{2}(t, r)\left|\int_{0}^{t}\left[v_{\tau}-\tilde{v}_{\tau}\right] d \omega\right|^{2} q(d \tau) d t \\
& \leq E \int_{0}^{T} e^{-\gamma \mathscr{K}(t)} \int_{0}^{t}\left|v_{\tau}-\tilde{v}_{\tau}\right|^{2} d \tau d t \leq(L / 3)^{2}\|v-\tilde{v}\|_{L^{2}}^{2},
\end{aligned}
$$

and

$$
E \int_{0}^{T} e^{-\gamma \mathscr{G}(t)} \int_{\mathbb{R}^{m}} m^{2}(t, r)\left|\int_{0}^{t} \int_{\mathbb{R}^{m}}\left[z_{\tau, r}-\widetilde{z}_{\tau, r}\right] \widetilde{\nu}(d \tau, d r)\right|^{2} q(d \tau) d t
$$




$$
\leq E \int_{0}^{T} e^{-\gamma \mathscr{K}(t)} \int_{0}^{t} \int_{\mathbb{R}^{m}}\left|z_{\tau, r}-\widetilde{z}_{\tau, r}\right|^{2} q(d r) d \tau d t \leq(L / 3)^{2}\|z-\widetilde{z}\|_{W^{2}}^{2} .
$$

Therefore,

$$
\begin{aligned}
& \ell_{\mathcal{W}^{2}}\left(S_{q}\left(H \circ \Phi^{\lambda}(u, v, r)\right), S_{q}\left(H \circ \Phi^{\lambda}(\tilde{u}, \tilde{v}, \tilde{z})\right)\right. \\
\leq & L \max \left(\|u-\tilde{u}\|_{\mathcal{L}^{2}},\|v-\tilde{v}\|_{\mathcal{L}^{2}},\|z-\tilde{z}\|_{W^{2}}\right.
\end{aligned}
$$

Now we can prove the following basic lemma.

Lemma 1. Suppose $F, G$, and $H$ satisfy $\left(\mathcal{A}_{1}\right)$ and $\left(\mathcal{A}_{2}\right)$. There is a norm $\|\cdot\|$ on $\mathcal{L}^{2} \times \mathcal{L}^{2} \times W^{2}$ equivalent to the norm defined on $\mathcal{L}^{2} \times \mathcal{L}^{2} \times \mathbb{W}^{2}$ by $|\cdot|_{\mathcal{L}^{2}}$ and $|\cdot|_{W^{2}}$ such that $S_{\lambda}(\cdot)$ and $S .(u, v, z)$ are contractions from $\left(\mathcal{L}^{2} \times \mathcal{L}^{2} \times W^{2},\|\cdot\|\right)$ and $\left(L_{n}^{2}\left(\mathscr{F}_{0}\right),\|\cdot\|_{L_{n}^{2}}\right)$, respectively, into $\left(\mathrm{Cl}\left(\mathcal{L}^{2} \times \mathcal{L}^{2} \times W^{2}\right), \ell\right)$, where $\ell$ is the Hausdorff metric induced by the norm $\|\cdot\|$.

Proof. Let $L \in[0,1)$ and $\|\cdot\|_{\mathcal{L}^{2}}$ and $\|\cdot\|_{W^{2}}$ be such as in Proposition 2, corresponding to the given $L$. Set $\|(u, v, z)\|=\max \left(\|u\|_{\mathcal{L}^{2}},\|v\|_{\mathcal{L}^{2}},\|z\|_{W^{2}}\right)$ and let $\ell$ be the Hausdorff metric on $\mathrm{Cl}\left(\mathcal{L}^{2} \times \mathcal{L}^{2} \times \mathcal{W}^{2}\right)$ induced by the norm $\|\cdot\|$. By Proposition 2, we obtain

$$
\ell\left(S_{\lambda}(u, v, z), S_{\lambda}(\widetilde{u}, \widetilde{v}, \widetilde{z})\right) \leq L\|(u, v, z)-(\tilde{u}, \tilde{v}, \widetilde{z})\|
$$

for $\lambda \in L_{n}^{2}\left(\mathscr{F}_{0}\right)$ and $(u, v, z),(\tilde{u}, \widetilde{v}, \widetilde{z}) \in \mathcal{L}^{2} \times \mathcal{L}^{2} \times \mathscr{W}^{2}$. Quite similarly,

$$
\ell\left(S_{\lambda}(u, v, z), S_{\tilde{\lambda}}(u, v, z)\right) \leq L\|\lambda-\tilde{\lambda}\|_{L_{n}^{2}}
$$

for $\lambda, \tilde{\lambda} \in L_{n}^{2}\left(\mathscr{F}_{0}\right)$ and $(u, v, z) \in \mathcal{L}^{2} \times \mathcal{L}^{2} \times W^{2}$.

\section{Quasi-Retractive Representation of Solution Set}

We shall show that if conditions $\left(\mathcal{A}_{1}\right)$ and $\left(\mathcal{A}_{2}\right)$ are satisfied, then the solution set mapping $\lambda \rightarrow \mathbb{C}_{\lambda}$, where $\mathfrak{C}_{\lambda}$ denotes a set of all solutions to an initial value problem $S I_{\lambda}(F, G, H)$, has quasi-retractive representation. In particular, it will follow that this mapping is lower semicontinuous. Moreover, it will follow that in some special cases the solution set $e_{\lambda}$ is weakly compact in $\left(D,\|\cdot\|_{\ell}\right)$. These results are consequences of Lemma 1 and a general retractive representation theorem presented in [1].

Let $\Lambda$ be a topological space and $(X,|\cdot|)$ be a Banach space. Denote $\mathcal{N}(X)=$ $\{A \subset X: A \neq \emptyset\}$. Given $S: \Lambda \rightarrow \mathcal{N}(X)$ and $C \subset X$, let $S^{-}(C)=\{\lambda \in \Lambda: S(\lambda) \cap C \neq \emptyset\}$. We say that $S: \Lambda \rightarrow \mathcal{N}(X)$ is lower semicontinuous (l.s.c.) [upper semicontinuous (u.s.c.)] if $S^{-}(C)$ is open [closed] for every open [closed] set $C \subset X$. A set-valued mapping $S: \Lambda \rightarrow \mathcal{N}$ is said to be $W$-upper semicontinuous ( $W$-u.s.c.) if for every $x \in X$ the function $\lambda \rightarrow \operatorname{dist}(x, S(\lambda))$ is lower semicontinuous in the usual sense. Finally, $S$ is said to be $W$-continuous if it is l.s.c. and $W$-u.s.c. 
We say that $S: \Lambda \rightarrow \mathcal{N}(X)$ has a retractive representation if there exists a set $B \in \mathcal{N}(X)$ and a continuous mapping $p: \Lambda \times B \rightarrow B$ such that $p(\lambda, x) \in S(\lambda)$ for every $(\lambda, x) \in \Lambda \times B$ and $p(\lambda, x)=x$ if and only if $x \in S(\lambda)$.

We say that the solution set mapping $L_{n}^{2}\left(\mathscr{F}_{0}\right) \ni \lambda \rightarrow \mathrm{C}_{\lambda} \subset D$ has quasi-retractive representation if there is a set-valued mapping $S: L_{n}^{2}\left(\mathcal{F}_{0}\right) \rightarrow \mathcal{N}\left(\mathcal{L}^{2} \times \mathcal{L}^{2} \times \mathcal{W}^{2}\right)$ having a retractive representation $p: \Lambda \times B \rightarrow B$ such that $\mathcal{C}_{\lambda}=\Phi^{\lambda}(p(\lambda, B))$, each $\lambda \in L_{n}^{2}\left(\mathscr{F}_{0}\right)$.

We present the following general results (see $[1,8]$ ) dealing with retractive representation of set-valued mappings.

Theorem 2. ([8], Th. 1) Let $\Lambda$ be a paracompact and perfectly normal topological space, $(X,|\cdot|)$ be a Banach space, and $B \in \mathrm{Cl}(X)$. Suppose $९: \Lambda \times B \rightarrow \mathrm{Cl}(X)$ takes on convex values and is such that:

(i) for every $x \in B$ the set-valued mapping $\mathscr{P}(\cdot, x)$ is $W$-continuous,

(ii) there is $L \in[0,1)$ such that $h(\mathscr{P}(\lambda, x), \mathscr{P}(\lambda, \tilde{x})) \leq L|x-\widetilde{x}|$ for fixed $\lambda \in \Lambda$ and $x, \tilde{x} \in B$, where $h$ is Hausdorff metric on $\mathrm{Cl}(X)$ induced by the norm $|\cdot|$.

Let $S_{\mathscr{\rho}}(\lambda):=\{x \in B: x \in \mathscr{P}(\lambda, x)\}$, each $\lambda \in \Lambda$. A set-valued mapping $S_{\mathscr{~}}: \Lambda \ni$ $\lambda \rightarrow S_{\text {gp }}(\lambda) \in \mathcal{N}(B)$ has a retractive representation $p: \Lambda \times B \rightarrow B$.

We now apply Theorem 2 and Lemma 1 to the subtrajectory integrals mapping $S$. defined above. Recall that for given $F, G$, and $H$ satisfying condition $\left(\mathcal{A}_{1}\right)$, we can define a convex, weakly compact set $B$ (see Corollary 1), where $B$ is a subset of a Banach space $\left(\mathscr{L}^{2} \times \mathcal{L}^{2} \times W^{2},\|\cdot\|\right)$ with a norm $\|\cdot\|$ defined in Lemma 1 corresponding to any $L \in[0,1)$, containing the set $C(\lambda)$ of all fixed points to subtrajectory integrals mapping $S_{\lambda}(\cdot)$. From Theorem 2 and Lemma 1 we immediately obtain the following result.

Lemma 3. Suppose $F, G$, and $H$ satisfy conditions $\left(\mathcal{A}_{1}\right)$ and $\left(\mathcal{A}_{2}\right)$. A set-valued mapping $C: L_{n}^{2}\left(\mathscr{F}_{0}\right) \ni \lambda \rightarrow C(\lambda) \in \mathcal{N}(B)$ has a retractive representation $p: L_{n}^{2}\left(\mathscr{F}_{0}\right) \times$ $B \rightarrow B$.

Corollary 2. Let $F, G$, and $H$ satisfy conditions $\left(A_{1}\right)$ and $\left(\mathcal{A}_{2}\right)$, and $p: L_{n}^{2}\left(\mathcal{F}_{0}\right) \times$ $B \rightarrow B$ be a retractive representation for $S$. Then $C(\lambda)=p(\lambda, B)$, each $\lambda \in L_{n}^{2}\left(\mathscr{F}_{0}\right)$.

Corollary 3. The set-valued mapping $\lambda \rightarrow C(\lambda)$ is continuous as a mapping from $L^{2}\left(\mathscr{F}_{0}\right)$ into a metric space $\left(\mathrm{Cl}\left(\mathcal{L}^{2} \times \mathcal{L}^{2} \times \mathcal{L}^{2}, \ell\right)\right.$.

Given $F, G$, and $H$ satisfying conditions $\left(\mathcal{A}_{1}\right)$ and $\left(\mathcal{A}_{2}\right), \mathcal{C}_{\lambda}$ denotes a set of all solutions to the initial value problem $S I_{\lambda}(F, G, H)$. As an immediate consequence of Proposition 1, we obtain $\mathrm{C}_{\lambda}=\Phi^{\lambda}(C(\lambda))$, where $C(\lambda)$ is defined as above. $\mathrm{C}$ denotes a set-valued mapping $L_{n}^{2}\left(\mathscr{F}_{0}\right) \ni \lambda \rightarrow \mathrm{e}_{\lambda} \subset D$. From the above definitions, Lemma 3 , and properties of $\Phi^{\lambda}$, we immediately obtain the following main result of this paper.

Theorem 4. If $F, G$, and $H$ satisfy conditions $\left(\mathcal{A}_{1}\right)$ and $\left(\mathcal{A}_{2}\right)$, then $\mathrm{C}$ has a quasiretractive representation and is l.s.c. on $L^{2}\left(\mathcal{F}_{0}\right)$.

Proof. Let $p: L_{n}^{2}\left(\mathscr{F}_{0}\right) \times B \rightarrow B$ be a retractive representation for the set-valued mapping $C$ defined in Lemma 3. We have $\mathcal{C}_{\lambda}=\Phi^{\lambda}(C(\lambda))$ and $C(\lambda)=p(\lambda, B)$, each $\lambda \in L_{n}^{2}\left(\mathscr{F}_{0}\right)$. Therefore, $C$ has a quasi-retractive representation. Moreover, a function $L_{n}^{2}\left(\mathscr{F}_{0}\right) \ni \lambda \rightarrow \Phi^{\lambda}(p(\lambda, x)) \in D$ is continuous for fixed $x \in B$. Therefore, a set-valued mapping $C$ (see [3], Proposition II 2.5) is l.s.c. on $L^{2}\left(\mathscr{F}_{0}\right)$.

Corollary 4. If $F, G$, and $H$ satisfy conditions $\left(\mathcal{A}_{1}\right)$ and $\left(\mathcal{A}_{2}\right)$ and are such that a set-valued mapping $C$ has a retractive representation $p: L_{n}^{2}\left(\mathscr{F}_{0}\right) \times B \rightarrow B$ that is weakly-weakly continuous, then $\mathcal{C}_{\lambda}$ is a weakly compact subset of $D$ for every $\lambda \in L_{n}^{2}\left(\mathscr{F}_{0}\right)$ and a set-valued mapping $\mathrm{C}$ is weak-weak continuous on $L_{n}^{2}\left(\mathscr{F}_{0}\right)$.

Proof. Indeed, if $p$ has properties mentioned above, then (see [3], Th. II 2.6) 
$p(\lambda, B)$ is a weakly compact subset of $B$ for each $\lambda \in L_{n}^{2}\left(\mathscr{F}_{\rho}\right) . \quad \mathrm{C}_{\lambda}$ is also a weakly compact subset of $B$ for each $\lambda \in L_{n}^{2}\left(\mathscr{F}_{0}\right)$ because $\mathcal{C}_{\lambda}=\Phi(p(\lambda, B))$. Finally, by weak-weak continuity of the linear mapping $L_{n}^{2}\left(\mathscr{\mathscr { F }}_{0}\right) \times B \ni(\lambda, x) \rightarrow \Phi^{\lambda}(x)$, weak compactness of $B$, and an equality $C_{\lambda}=\Phi^{\lambda}(p(\lambda, B))$, each $\lambda \in L_{n}^{2}\left(\mathscr{F}_{0}\right)$, it follows (see again [3], Proposition II 2.5), that $\mathcal{C}$ is weak-weak continuous on $L_{n}^{2}\left(\mathscr{F}_{0}\right)$.

\section{References}

[1] Drewnowski, L., Kisielewicz, M. and Rybiński, L., Minimal selections and fixed points of multivalued contraction mappings in uniformly convex Banach space, Comm. Math. 29 (1989), 43-50.

[2] Hiai, F. and Umegaki, H., Integrals, conditional expectations and martingales of multifunctions, J. Multivalued Anal. 7 (1977), 149-182.

[3] Kisielewicz, M., Differential Inclusions and Optimal Control, Kluwer Acad. Publ. and Polish Sci. Publ., Warszawa-Dordrecht-Boston-London 1991.

[4] Kisielewicz, M., Properties of solution set of stochastic inclusions, J. of Appl. Math and Stochastic Analysis 6:3 (1993), 217-236.

[5] Kisielewicz, M., Set-valued stochastic integrals and stochastic inclusions, Stoch. Anal. and Appl. (to appear).

[6] Markin, T.J., Stability of solution sets for generalized differential equations, J. Math. Anal. Appl. 46 (1974), 289-291.

[7] Protter, Ph., Stochastic Integration and Differential Equations, Springer-Verlag, Berlin-Heidelberg-New York 1990.

[8] Rybiński, L., A fixed point approach in the study of solution sets of Lipschitzian functional-differential inclusions, J. Math. Anal. Appl. 160 (1991), 24-46. 


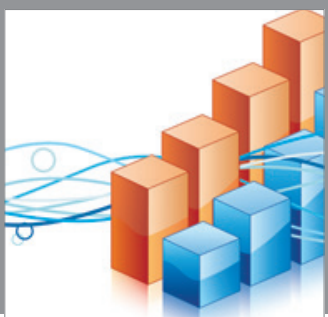

Advances in

Operations Research

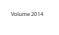

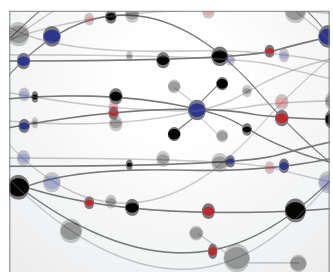

\section{The Scientific} World Journal
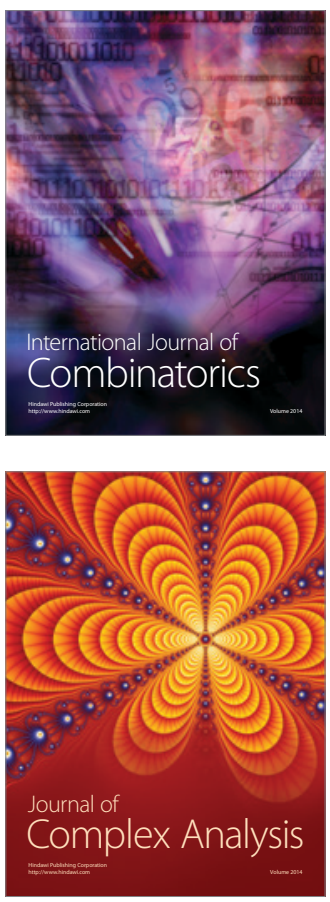

International Journal of

Mathematics and

Mathematical

Sciences
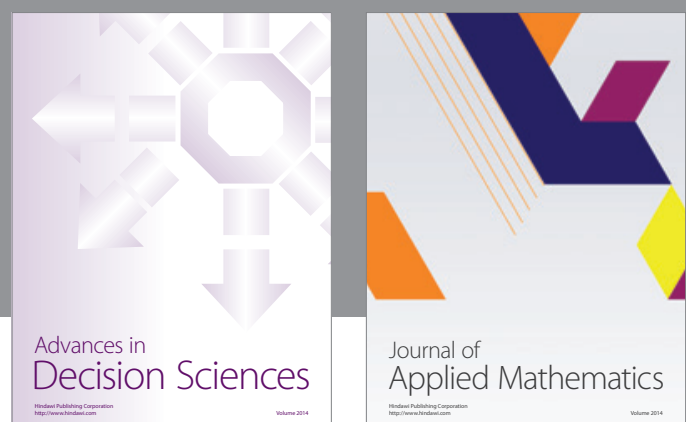

Journal of

Applied Mathematics
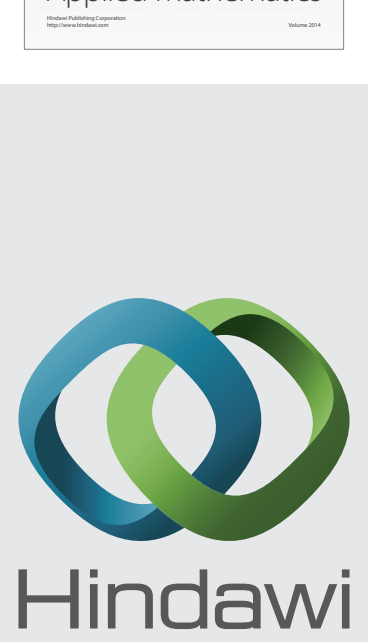

Submit your manuscripts at http://www.hindawi.com
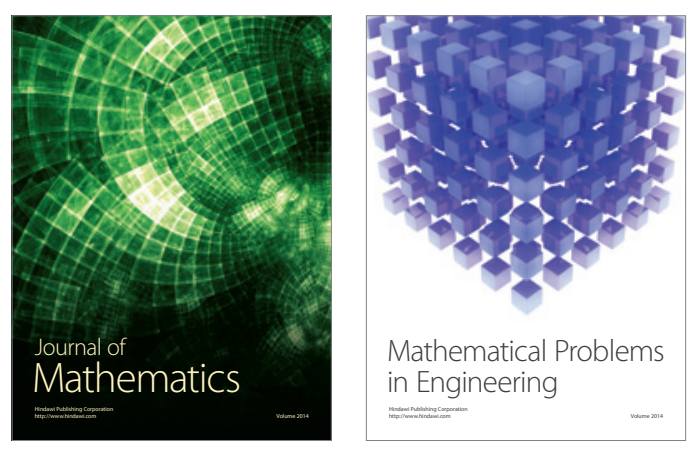

Mathematical Problems in Engineering
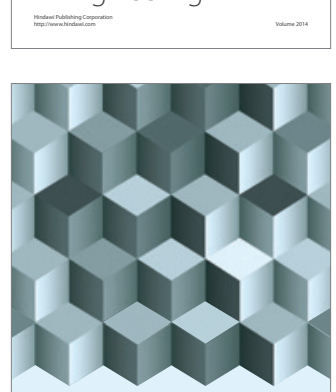

Journal of

Function Spaces
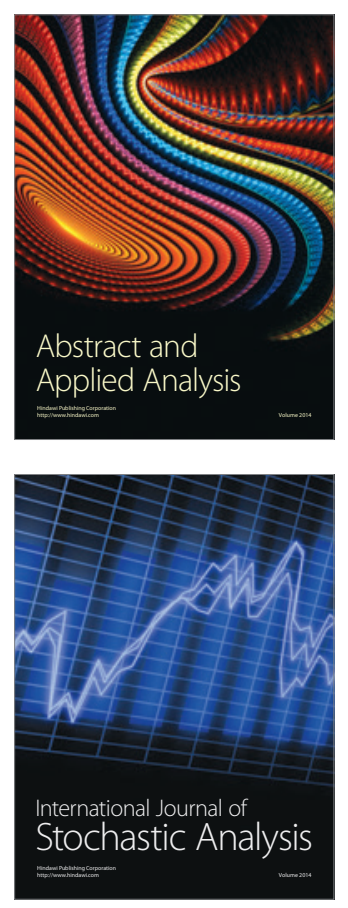

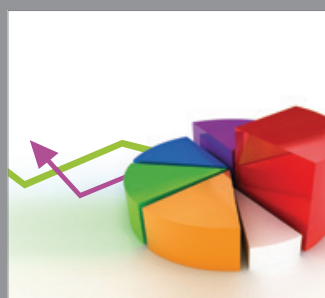

ournal of

Probability and Statistics

Promensencen
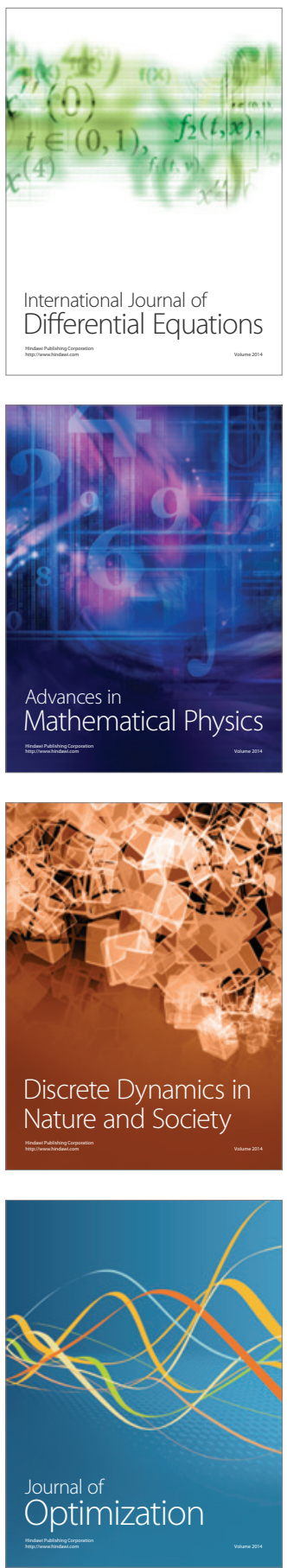